\title{
GATINEAU SCALE RATIOMETER FOR USE WITH VERTICAL PHOTOGRAPHS IN DETERMINING SCALE AND RATIO
}

\author{
By F. R. Wricox \\ Prepared at Maniwaki, P. Q., March, 1936
}

$\mathbb{T}$

7 HE SCALE ratiometer is a simple measuring device designed essentially to facilitate the use of individual contact prints in the field as they are used by foresters and field engineers.

The first necessity in applying aerial photographs to map making is a knowledge of the ratio of distance between image points on the photographs and the corresponding distance between the actual objects on the ground, in other words the scale of the photograph.

The scales of different sets of photographs, different individual photographs and even different sections of the same photograph may differ.

Much has been written on the theory of map preparation from aerial photographs. There are several accepted and standard map making procedures, each with numerous variations, all of which have been analyzed by various

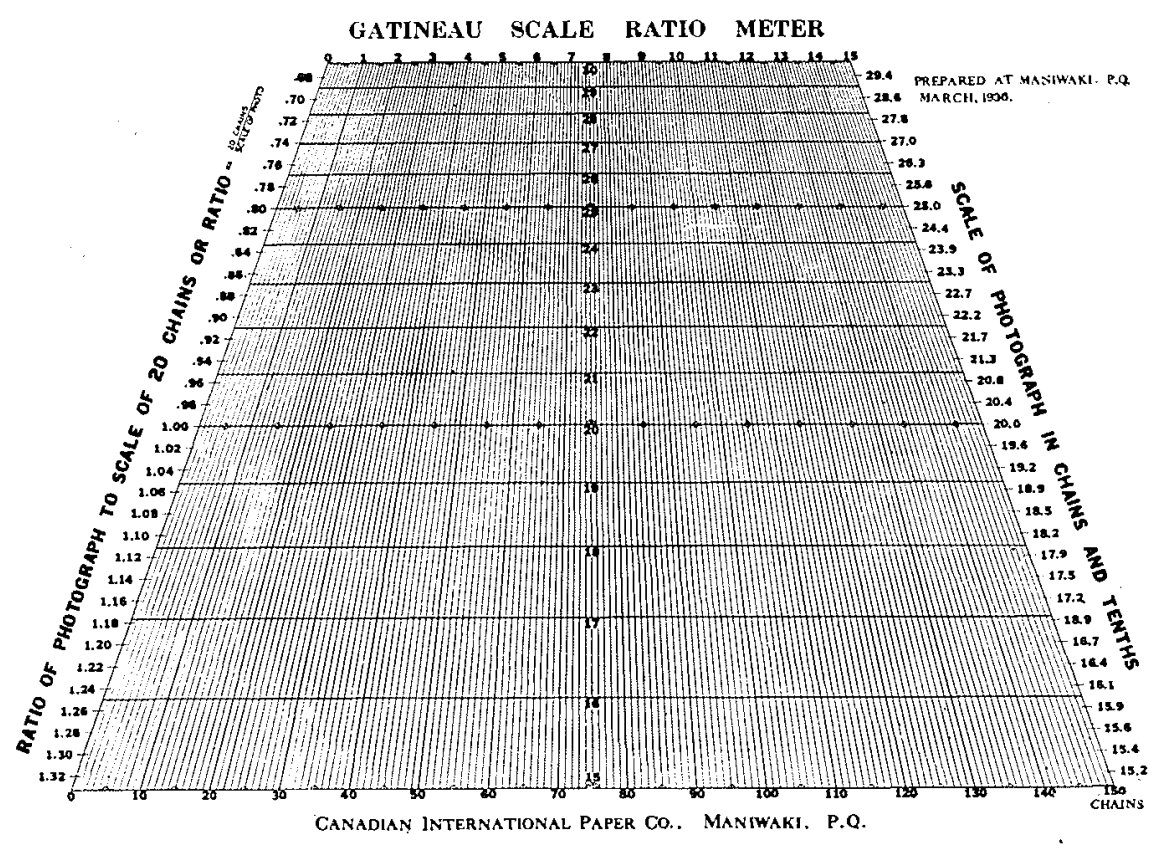


writers in current technical magazines (see article by F. R. Wilcox, in Forestry Chronicle).

To the knowledge of the writer there has been little, if any, written material prepared which offers guidance and help to the field man attempting to put the photographs to practical use in the field after the map has been prepared.

The theory behind the most used method (radial line) of producing an aerial map provides for a constant scale being adopted for all photographs in any one flight, or section of flight, between controls. The scale so adopted originates from the first picture of the flight and should have as its origin ground control. Starting from this origin, the method then carries this scale forward and applies it to all subsequent photographs in the flight until the next control is reached.

As the mapping operation proceeds over an area, each flight has a given scale which may differ from any other flight and the scale of all flights will undoubtedly differ from the scale of the final map. The various scales, of course, are brought to one common scale.

Finally when the finished map is delivered to the field man he has a plan which may look like Figure. 1. This plan shows the planimetry and the principle point traverse.

To get the most out of any aerial map the contact prints must be used by the field men who are working with the map. This is fine, we have handed our field man a map and a set of contact prints. The map of course, has a common scale throughout but the contact prints in which we are placing so much faith and with which we tell our field staff they can do wonders, contain a very peculiar conglomeration of scales.

Even though the photography was carried out with a combination of altitude and focal length of lens to produce in theory, pictures at the scale of the final map, these same scales as they actually occur on the photograph are likely and justificably so, to vary $10 \%$. Then there is the question of tilt and configuration of the terrain which again adds to the worries of our contact print user.

When finally all the scale influencing factors have been analysed we find that our field man has a set of contact prints, any one print of which may contain planimentry, represented by several different scales, to say nothing of the scale discrepancies occurring on various prints. Such scale differences are usually small but may be large and in all cases are troublesome to the field man.

In summarizing the scale situation we find that, the most significant scale difference is that which occurs between the theoretic scale of the pictures and the scale of the final map which is additionally confusing when it is borne in mind that all other things being equal, there is likely to be an appreciable scale 


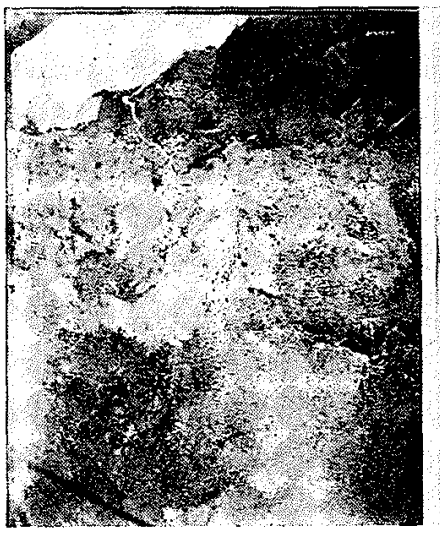

(a)

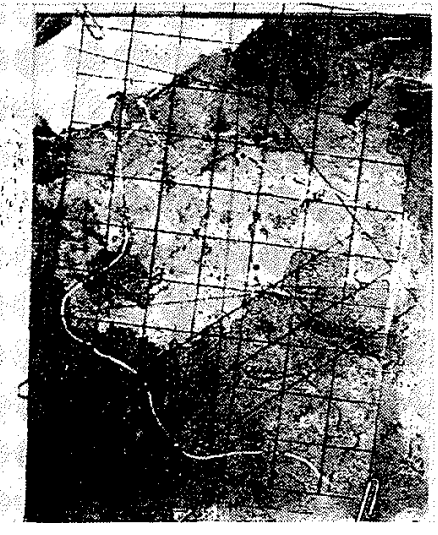

(b)

differential between any two contact prints due to change in altitude of the air stations.

So much for the cause, here is a remedy. The Gatineau scale ratiometer as shown in Figure 2. is printed on transparent celluloid, lumarith or glass. The particular one shown has been developed for a base map scale of 20 chains to the inch. It is used thus:

1. To determine the scale of any contact print or section of print.

(a) In relation to Scale of Map-Measure on the centre, horizontal, even 20 scale line the distance on the 4 " map between any two features that may be readily identified on the photograph. Assume, for example, the distance so scaled to be 60 chains. Superimpose the scale ratiometer upon the contact print, making the centre horizontal line lay along the course to be measured. Unless the picture is exactly 20 chains to the inch, this distance will be found to be greater or less than sixty chains: now slide the ratiometer up or down until it reads 60 chains between the two points. When this correspondence is established, follow the horizontal to the right hand margin and read the scale of the picture, in relation to the 4" Base Map, direct.

(b) In relation to Distance determined on the Ground-The procedure is the same as above with the exception that the distance measured on the map is now measured on the ground. The measurcment may be paced, chained, by stadia, by counting the revolutions of a buggy wheel, motor car speedometer or guessed according to the accuracy desired and the inclination of the individual. The resultant scale determincd for the picture, will be just as accurate as the ground measurement made.

The utilization of the scale ratiometer is two fold. As mentioned above 
the scale is read by following the horizontal line to the right. Follow the same line to the left and the percentic relation between the scale of the photograph and the scale of the map may be read direct.

This ratio is particularly valuable to the forester using photographs in the field, under office tent conditions that do not allow for the use of an optical projector for transferring picture information to the base map.

When pictures are used to facilitate cruising the most desirable procedure is to carry the contact prints in the field for the purpose of checking previous office interpretation and to identify cruise line ties as often as possible along the cruise line route. The same condition exists when running lines for the establishment and location of spot heights in contour work.

All such information must be transferred to the base map after each days work.

A practical and economically procedure for making this transfer is the employment of proportional grids. These grids consist of squares etched on celluloid or lumerath. (See Figure 3) For accuracy of the order required for forest maps a grid for each .02 of ratio is found to be sufficient.

The procedure in using the ratiometer and also the grids in transferring picture information is as follows:

A cruise line has been run. It is desired to plot it on the contact print, complete the final picture interpretation and transfer all this information to the hase map. (By base map is meant a planimetric map of the main features as shown in Figure 1.) In plotting the cruise line the scale of the line location, on the picture is determined by using the scale ratiometer as described under Item 1-b. The same operation determines the ratio of the photograph. The corresponding grid for this ratio is then superimposed upon the photograph (see Fig. 3) and the detail is transferred to the base map by proportional squares. It will be noted from a study of figure 1, that squares have been ruled on the base map for this purpose.

This ruling is done in yellow and does not interfere with future cartographic work on the map.

The range of usefulness of the ratiometer can be extended one hundred percent in each direction by doubling all figures or reducing them by $50 \%$. However, if such a range is required constantly, it would be advisable to make the two additional projections.

The scale ratiometer is simply made. The particular one illustrated in Figure 2 was drafted at three times its desired final dimensions. This projection was then reduced by the wet plate process and finally printed upon celluloid or glass. One very useful model has been printed upon celluloid $1 / 20^{\prime \prime}$ thick (about the thickness of the regular drafting-room triangle) and bevelled slits cut along the even chain scale lines so that the instrument can be used to mark scaled distances. 